\title{
Craving e dependência química: conceito, avaliação e tratamento
}

\author{
Craving and chemical dependence: concept, evaluation and treatment
}

Renata Brasil Araujoํ, Margareth da Silva Oliveira', Rosemeri Siqueira Pedroso', Alessandra Cecília Miguel', Maria da Graça Tanori de Castro'

\section{RESUMO}

O craving ou fissura, cuja definição mais comum é o desejo intenso por uma substância, é um conceito controverso entre os pesquisadores da área da dependência química. Objetivo: Realizar revisão teórica a respeito do craving nos bancos de dados Psyclnfo, Medline, ProQuest e Science Direct. Método: As palavras-chave utilizadas foram craving, dependence e drug e o período pesquisado foi entre 1995 e 2007. Resultados: Os resultados demonstraram que são encontrados diversos significados para o craving, alguns se restringindo a desejo, e outros, considerando-o não só como desejo, mas como antecipação do resultado positivo do uso da substância, alívio dos sintomas de abstinência ou afeto negativo e intenção de fumar, o que reflete uma visão multidimensional deste construto. A etiologia do craving pode ser explicada por intermédio dos modelos: comportamental, cognitivo ou psicossocial e neurobiológico, porquanto a opção por um destes influencia a avaliação e

\section{Palavras-chave}

Craving,

dependência química, avaliação, tratamento.

o manejo. Conclusão: Conclui-se quanto à multidimensionalidade do craving e quanto à necessidade de que seja utilizado um modelo biopsicossocial que integre os diversos modelos no tratamento de dependentes químicos. Destaca-se a importância da realização de mais estudos para a compreensão do craving em função deste ser um dos principais riscos de recaída.

\section{ABSTRACT}

Craving is usually defined as an intense desire for a substance. This is a rather controversial concept among the researchers on the chemical dependence area. Objective: The objective of this study was to carry out a theoretical review concerning craving at Psyclnfo, Medline, ProQuest, Portal Capes and Science Direct Data Bases. Methods: The keywords used were craving, dependence and drug, and the searching period was between 1995 and 2007. Results: The results showed that many different meanings can be found for craving, some relate it only to desire, while others consider it not only as desire, but also as the anticipation of the positive result from the use of the substance, the relief of abstinence symptoms or negative affect and intention to smoke, which reflects a multidimensional view of this construct. The ethimology of craving can be explained by

1 Pontifícia Universidade Católica do Rio Grande do Sul (PUCRS).

Recebido em

29/10/2007

Aprovado em $6 / 3 / 2008$

Endereço para correspondência: Renata Brasil Araujo

Av. Venâncio Aires, 1092, apto. 73 - 90040-192 - Porto Alegre, RS

Email: renataudbrasil@terra.com.br 


\section{Key-words}

Craving, chemical dependence, evaluation, treatment. the following Models: Behavioral, Cognitive or Psychosocial and Neurobiological, being the option for one of them a factor that influences evaluation and usage. Conclusion; As a conclusion, we are in favor of craving multidimensionality, as well as the need to use a Biopsychsocial Model that integrates the various models for treating chemical dependent patients. It is also necessary to highlight the importance of carrying out more studies in oder to understand craving, for it is one of the principal risks of relapse.

\section{INTRODUÇÃO}

O craving ou "fissura" - como é designado, popularmente, pelos dependentes químicos no Brasil - é um conceito um tanto controverso. Pode-se aceitar a definição mais comum e considerar que é um intenso desejo de utilizar uma específica substância ${ }^{1-4}$, ou, então, concordar com outros vários conceitos descritos pelos pesquisadores deste tema: desejo de experimentar os efeitos da droga; forte e subjetiva energia; irresistível impulso para usar droga; pensamento obsessivo; alívio para os sintomas de abstinência; incentivo para auto-administar a substância; expectativa de resultado positivo; processo de avaliação cognitiva e processo cognitivo não-automático ${ }^{5-7}$.

Estas divergências conceituais juntamente com a importância do entendimento do craving na clínica do dependente químico sugerem a necessidade de que sejam realizadas mais pesquisas a respeito deste construto.

Assim, o objetivo deste estudo é realizar uma revisão teórica a respeito do craving relacionado à dependência química e sua evolução, tanto em termos de pesquisa quanto da clínica, salientando as questões conceituais, os modelos teóricos que o fundamentam e algumas formas de avaliação e manejo. Optou-se pela utilização do termo craving, e não "fissura", por este último ser popularmente usado pelos dependentes químicos e sofrer influências regionais, não sendo uma nomenclatura plenamente aprovada no meio acadêmico.

\section{MÉTODO}

Este artigo de revisão foi elaborado a partir de pesquisa nos bancos de dados Psyclnfo, Medline, ProQuest e Science Direct. As palavras-chave utilizadas foram craving, dependence e drug e o período pesquisado foi o compreendido entre 1995 e 2004. Também foram analisados livros e artigos que se encontravam nas referências bibliográficas das fontes indexadas.

\section{A definição do craving}

A Organização Mundial de Saúde (OMS) reuniu um comitê de especialistas em dependência química que definiu o craving como um desejo de repetir a experiência dos efeitos de uma dada substânciå̊.

Este desejo pode ocorrer tanto na fase de consumo quanto no início da abstinência, ou após um longo tempo sem utilizar a droga $a^{3,9}$, costumando vir acompanhado de alterações no humor, no comportamento e no pensamento ${ }^{4,10}$.

O conceito mais utilizado de craving, como já foi enfatizado, é o que se refere a um intenso desejo para consumir determinada substâncial-3, porém pode ser mais amplamente definido como o reflexo de um estado de motivação orientado para o consumo de drogas?, conceito este que integra a idéia de desejo com utilização da substância.

O craving pode ser classificado em quatro tipos: como resposta à síndrome de abstinência; como resposta à falta de prazer; como resposta condicionada a estímulos relacionados às substâncias psicoativas; e como tentativa de intensificar o prazer de determinadas atividades ${ }^{1,11}$. Obviamente, é fácil perceber, que uma mesma situação pode fazer parte de mais de um destes grupos, tendo múltiplos determinantes.

Segundo Marlatt e Gordon'12, o craving é um estado motivacional subjetivo influenciado pelas expectativas associadas a um resultado positivo, estado este que pode induzir uma resposta na qual o comportamento desejado esteja envolvido.

Outro caminho na evolução do entendimento deste construto define o craving como um termo que engloba não somente o desejo, como a intenção de realizar este desejo, a antecipação dos efeitos positivos associados a sua utilização e o alívio do afeto negativo e dos sintomas relacionados à abstinência ${ }^{4,10,13}$.

Kozlowski et al. ${ }^{2}$ argumentam que as expectativas de resultado quanto aos efeitos, a intenção de consumir e o craving pela droga não são fatores que podem ser inseridos em uma mesma definição. Apesar de estes fatores demonstrarem estar correlacionados em vários estudos ${ }^{2,10}$, são conceitualmente diferentes, sendo, as expectativas e a intenção desencadeantes do craving².

Independentemente desses fatores fazerem parte ou serem causadores do craving, é importante definir quais foram as situações que estimularam o craving para que sejam mais bem planejadas as estratégias de prevenção de recaí- 
da'2, pois apesar de o craving estar associado com a recaída, não há determinação quanto a este desfecho', sendo possível que seja trabalhado o controle individual no manejo desta situação de risco.

\section{Modelos teóricos}

Existem vários modelos que explicam o craving: o comportamental, o psicossocial ou cognitivo e o neurobiológico, o que serve como evidência de sua multidimensionalidade e torna adequado que o seu tratamento seja proposto utilizando diversas referências ${ }^{3}$.

\section{O modelo comportamental}

O modelo comportamental foi o primeiro modelo descrito e tem como fundamentação o condicionamento clássico que explica a manutenção do uso de drogas como decorrência da expectativa de efeito de prazer aprendido a partir de experiências anteriores. A indução do craving pode, neste caso, ser entendida como reflexo de respostas condicionadas estabelecidas pela aprendizagem da associação entre determinado estímulo e o prazer que acompanha a resposta de usar a droga ${ }^{3,14}$.

A própria disponibilidade da substância tem sido associada ao aumento do craving $9,15,16$, assim como estímulos externos e a rede social relacionados ao uso de drogas ${ }^{17-19}$, sendo estes fatores importantes para o entendimento da origem do craving.

\section{O modelo psicossocial ou cognitivo}

O modelo psicossocial ou cognitivo explica o craving a partir da expectativa antecipada que o indivíduo tem quanto aos efeitos da substância, sendo salientada, ao contrário do modelo comportamental, a interpretação cognitiva feita a respeito deste fenômeno e não diretamente as respostas fisiológicas a ele relacionadas ${ }^{3}$. Tem, como fundamento, aspectos da Teoria do Aprendizado Social de Bandura ${ }^{20}$, que preconiza que a ocorrência de certo comportamento sofre a influência das expectativas que se tem do resultado desta ação, do valor que este resultado trará e da situação psicológica em que este comportamento acontece. Por outro lado, se o indivíduo sente-se capaz de manejar corretamente um comportamento em uma situação específica, ocorre o que é denominado de expectativa de auto-eficácia' ${ }^{12,21}$, visto que esta, no caso de dependentes químicos, varia de acordo com fatores como emoções positivas e negativas, situações de restrição ao uso da droga, reuniões sociais, horários ociosos, falta de prazer e craving ${ }^{22,23}$. Aspectos psicológicos, como estresse ${ }^{24,25}$, variáveis da personalidade ${ }^{26}$, aspectos motivacionais ${ }^{27,28}$, processos cognitivos automáti$\cos$ e não-automáticos ${ }^{28}$, referentes à memória dos efeitos das drogas ${ }^{29}$ e às expectativas de resultado destes efeitos ${ }^{30}$, contribuem para a etiologia do craving ${ }^{27}$, não devendo estes serem negligenciados pelos pesquisadores e terapeutas da área da dependência química.

\section{O modelo neurobiológico}

Em pesquisas com ratos, Epping et al. ${ }^{31}$ observaram diminuição da função do sistema de recompensa cerebral nestes animais quando em abstinência e relacionam esta alteração como um importante fator motivacional que contribui para o craving, para a continuidade do uso da droga e para o aumento das recaídas em humanos. Os sintomas de abstinência parecem estar positivamente correlacionados com o craving ${ }^{32,33}$, tanto que a intensidade deste diminui após as duas primeiras semanas sem o uso da substância ${ }^{34}$. Nesse caso, o craving pode ser descrito como um fenômeno de hipersensibilização que ocorre na falta da droga ${ }^{35}$.

Têm sido estudadas as relações entre o craving em dependentes de substâncias psicoativas e fatores genéticos ${ }^{8,18}$. Dependentes do tabaco, por exemplo, com história familiar de tabagismo experimentam maior intensidade de craving em situação de estresse do que aqueles que não têm esta história familiar ${ }^{25}$.

Alguns autores, ao apresentarem estímulos relacionados às drogas, observaram aumento do craving ${ }^{14} \mathrm{e}$ ativação das estruturas mesocorticolímbicas ${ }^{35}$. Investigações de como as mudanças em vários sistemas de neurotransmissão, como dopamina, serotonina, opióides, glutamato e noradrenalina, variam com o comportamento de procura pelas drogas, refletem o quanto é importante o estudo deste mecanismo neurobiológico para o entendimento do craving ${ }^{8}$.

Pode-se destacar a função da dopamina no comportamento de procura pela droga, em virtude da sua propriedade indutora de reforços positivos no centro de recompensa cerebral e de sua relação com o craving ${ }^{26,34}$, sendo este neurotransmissor ativado durante o consumo de substâncias psicoativas ${ }^{34}$. Deve ser salientada, também, a função da serotonina, por causa da sua influência na liberação dopaminérgica ${ }^{8}$. Há indicações de que a diminuição de serotonina seria uma das responsáveis pela ocorrência de craving em alcoolistas ${ }^{36}$ e em dependentes de cocaína ${ }^{37}$.

O uso de substâncias psicoativas está relacionado à ativação da área tegmental ventral, núcleo acumbens, amígdala, cíngulo anterior e córtex pré-frontal|17,38. A dependência de drogas induz à neuroadaptação cerebral, que altera a memória de estímulos associados ao comportamento de usar a droga ${ }^{38}$. Regiões cerebrais associadas à excitação, comportamentos compulsivos, memória e integração de estímulos sensoriais são ativadas durante a exposição a estímulos relacionados à droga, os quais, por sua vez, estimulam o craving ${ }^{17}$. As especificidades de cada substância psicotrópica e as características individuais dos dependentes químicos podem ser alguns dos determinantes da forma e da intensidade com os quais o craving se apresenta ${ }^{8,9}$. 


\section{A avaliação do craving}

A divisão da natureza do craving em diversas direções conceituais resulta desacordo não só quanto ao seu significado como quanto à sua forma de avaliação ${ }^{6,39}$ : certos pesquisadores questionam o craving ocorrido em dias ou semanas específicos ${ }^{1,40}$, porém, outros entendem o craving como um estado passageiro, $0^{9,0,13,41,42}$ que persiste por apenas alguns minutos 9 , sendo, portanto, impossível de ser verificado por longo período, haja vista as suas flutuações no decorrer de um mesmo dia ${ }^{4}$.

O craving pode estar ocorrendo independentemente de a pessoa ter consciência disso ${ }^{4}$, o que significa que medidas não-verbais (como a procura pela droga ou o seu consumo), medidas psicofisiológicas, neurobiológicas, medidas cognitivas ou expressões comportamentais (como as expressões faciais) ${ }^{43}$ são medidas importantes na avaliação do craving ${ }^{2,4}$.

Também podem ser observadas controvérsias, quanto à forma de mensuração do craving, causadas pelos diferentes significados a ele atribuídos: pode ser avaliado a partir de um único fator, como "desejo pela substância"2 ou como um construto multifatorial ${ }^{6,10,13,41,42}$. O craving, a partir do seu entendimento, como desejo pode ser verificado de várias formas pelo terapeuta: pelo relato espontâneo feito pelo cliente de alguma situação que lhe causou vontade de usar drogas, por meio de seus sonhos ${ }^{15,44}$, pelo registro diário dos pensamentos disfuncionais, no qual são descritos aspectos relacionados aos episódios de craving ${ }^{1}$, e da análise de autorelatos da intensidade do craving? ${ }^{2}$.

Medidas objetivas incluem um ou dois itens em escalas likert, escalas analógico-visuais ou sistemas computadorizados, bem como questionários específicos para avaliar o craving 2,6,9,10,40-42, visto que alguns destes instrumentos contemplam a multidimensionalidade deste construto ${ }^{10,13}$.

É preciso destacar que as medidas de craving, tanto uni quanto multidimensionais, que se referem a qualquer período que não o momento da avaliação (retrospectivas), sofrem o viés da memória, não sendo totalmente confiáveis ${ }^{4}$. Anton e Drobes $^{39}$ indicam que, muitas vezes, na avaliação do craving não é considerada a validade dos instrumentos, nem são observadas as características específicas do craving de acordo com a substância psicoativa a qual se relaciona, havendo, nestes casos, claro prejuízo à fidedignidade de sua mensuração.

\section{O manejo do craving}

À medida que o craving tem sido considerado fator relevante na manutenção da abstinência, torna-se imprescindível o estudo de seu manejo para que se possa ser bem-sucedido no tratamento de dependentes químicos ${ }^{3}$.

O craving é, segundo Knapp, Luz Júnior e Baldisserotto"1, uma experiência individual, necessitando, portanto, de pla- nejamento personalizado de estratégias para o seu manejo. Essas técnicas de manejo do craving, provenientes da terapia cognitivo-comportamental, devem ser ensinadas desde o início do tratamento, sendo um importante instrumento terapêutico. Algumas das principais técnicas, citadas por Knapp; Luz Júnior; Baldisserotto"1 são:

\section{Distração}

Propõe-se que o indivíduo desloque a atenção das suas sensações e dos pensamentos relacionados ao craving para o mundo externo (por exemplo, fazendo alguma atividade).

\section{Cartões de enfrentamento}

São fichas portáteis com frases de impacto que servem para motivar o sujeito para que não use drogas. Pode conter as desvantagens de usar, as vantagens em estar em abstinência ou sentenças automotivacionais.

\section{Relaxamento}

São treinadas, na consulta, as técnicas de relaxamento, tanto por meio da respiração quanto da distensão muscular.

\section{Refocalização}

O indivíduo deve focalizar seu pensamento em uma frase como "Pare!" ou em uma imagem específica não compatível com o uso da droga.

\section{Substituição por imagem negativa}

O cliente deve substituir uma imagem positiva que estaria associada ao uso da droga por outra, negativa ligada aos prejuízos.

\section{Substituição por imagem positiva}

Deve ser feita uma visualização de si próprio como um vencedor que adquire benefícios a partir da interrupção do uso da droga.

\section{Ensaio por visualização}

Utilizado para preparar o indivíduo para enfrentar situações de risco. O dependente químico deve, guiado pelo terapeuta, imaginar-se em uma situação perigosa agindo de uma forma assertiva, sem utilizar a substância, sendo trabalhados os pensamentos e as emoções desencadeados, até que ele sinta-se seguro para enfrentá-la.

\section{Visualização de domínio}

É utilizada quando o cliente não consegue se imaginar resistindo ao craving. Ele é auxiliado pelo terapeuta a imaginarse vencendo a situação geradora de ansiedade, devendo ser quem decide os rumos da ação fantasiada.

Outras estratégias, como a realização de exercícios físi$\cos ^{45}$ e a automassagem ${ }^{46}$, têm obtido resultados satisfatórios para a redução do craving e dos sintomas de abstinência. 
O uso de psicofármacos para o manejo do craving tem sido alvo de várias pesquisas ${ }^{47-52}$, no entanto, poucos agentes têm provado ser efetivos no controle do craving, havendo limitações em seus efeitos ${ }^{53}$, sendo necessário que sejam realizadas mais pesquisas neste sentido.

A prescrição de psicofármacos não pode, portanto, excluir o uso das técnicas de prevenção da recaída, bem como de outras técnicas de manejo do craving: os ensaios clínicos realizados indicam que a combinação da farmacoterapia com o tratamento psicoterápico é a terapêutica que traz os melhores resultados para os dependentes químicos ${ }^{49,54}$.

\section{DISCUSSÃO}

Ao analisar esta diversidade conceitual, não há como deixar de pensar que o desejo só pode ser entendido em sua relação com o objeto desejado e com as conseqüências desta realização. Esses fatores parecem se misturar quando se avalia o craving de um dependente químico por determinada substância. O entendimento deste construto como multidimensional ${ }^{3,10,13,41,42}$ torna mais fácil de ser compreendida a dificuldade que os indivíduos relatam quanto ao seu controle.

Os modelos comportamental e psicossocial ou cognitivo poderiam também ser vistos como um modelo único, dado o seu evidente caráter complementar. A díade estímuloresposta do condicionamento clássico passa pela filtragem das crenças, expectativas ou mesmo restrições individuais, quando tratamos do uso de substâncias entorpecentes, o que foi descrito por vários autores ${ }^{20-22,26-28,30}$. Mesmo que a utilização de uma droga tenha como efeito direto o prazer, trará outras conseqüências não tão positivas que fazem que seja criado um dilema quanto à repetição deste comportamento, sendo justamente este espaço entre o desejo e sua realização um dos focos terapêuticos na área da dependência química.

Por outro lado, como as substâncias psicoativas têm ação neuroquímica ${ }^{8,17,26,31-38}$ que está relacionada não somente com o efeito das drogas, como com diversas outras alterações, tanto cognitivas quanto comportamentais, estes mecanismos precisam ser estudados para a compreensão do craving.

O modelo neurobiológico é bastante valorizado no tratamento da dependência química, até porque tanto o uso quanto a síndrome de abstinência das drogas vêm acompanhados de uma série de alterações neuroquímicas que, por sua vez, modificam e são modificadas por fatores não só de ordem física, como psicológica ${ }^{44}$. O craving, analisado sob este ponto de vista, também tem a sua etiologia associada ao modelo neurobiológico, havendo a necessidade destes aspectos, não isoladamente, mas de maneira integrada com os demais modelos, serem contemplados quando se propõe a sua avaliação.

O delineamento da avaliação do craving, sem dúvida alguma, será influenciado pelo que se considera como fazendo parte desse construto, idéia essa também compartilhada por Anton e Drobes ${ }^{39}$, assim como por Singleton et al. ${ }^{6}$. A multicausalidade do craving $^{3}$ parece apontar a necessidade de uma avaliação o mais completa possível para o entendimento desse fenômeno, no entanto, em alguns momentos, na clínica do dependente químico, é útil o uso de instrumentos de fácil aplicação e levantamento que sejam capazes de orientar estratégias de prevenção da recaída e de automanejo do craving.

Pode-se concluir que não existe uma única forma de mensurar o craving que sirva para todas as situações ${ }^{4}$, entretanto são necessárias novas pesquisas para que as medidas de avaliação do craving possam oferecer subsídios para a melhor definição deste construto, bem como dos fatores a este relacionados.

É possível observar que as técnicas de manejo do craving pesquisadas e utilizadas na prática clínica ${ }^{11,18,19,27,45,46,53-59}$ provêm de diversas fundamentações, podendo ser categorizadas como comportamentais, cognitivas ou psicofarmacológicas. Percebe-se que, muitas vezes, é necessária a integração de várias dessas técnicas para controlar o craving, conforme preconizam Johnson et al. ${ }^{49}$ e Baker ${ }^{54}$, e, que, mesmo assim, nem sempre se obtém sucesso nesse intuito.

Ainda não foi descoberto, apesar de todos os avanços, um psicofármaco totalmente eficaz para o manejo do craving das diferentes substâncias psicoativas ${ }^{53}$, porém percebe-se o interesse dos pesquisadores em encontrar alternativas neste sentido.

Enquanto isso não ocorre, a instrumentalização do dependente químico com várias técnicas para o manejo do craving parece ser o melhor caminho. Não é possível esquecer que o aumento da auto-eficácia dos clientes está relacionado à intensidade do desejo pela droga e, em decorrência disso, quando se prepara alguém para lidar com as situações de risco se está, de alguma forma, interferindo na intensidade do craving e tornando mais fácil o seu manejo.

Estudar a respeito do significado do termo craving é, sem dúvida alguma, tarefa difícil, cujo resultado não alcançará consenso entre os diversos estudiosos deste tema. Os conceitos de craving que concordam com uma visão unidimensional e que o entendem como "um desejo intenso de usar uma dada substância", sem associar este desejo com a intenção quanto ao consumo da droga e com as expectativas que se tem a respeito de seus efeitos reforçadores positivos e negativos, conseguem englobar apenas uma parte deste fenômeno.

Não é possível concordar com o posicionamento teórico que defende que expectativas e intenção não se relacionam ao próprio desejo, mas sim ao motivo de sua ocor- 
rência. A própria intenção de fumar subentende a idéia da intensidade deste desejo, bem mais do que sua causa.

$\mathrm{Na}$ verdade, quando se discute a respeito de desejo, não há como dissociá-lo de sua realização ou da crença que se tem a respeito do que esta realização trará como resultado. É claro que é possível que ocorra o craving por uma determinada substância sem que haja a intenção de utilizála - como é o exemplo de pessoas que estão tentando se manter em abstinência -, porém isso também esclarece, em parte, quanto a intensidade deste desejo.

Estas contradições conceituais que são, de certa forma, oriundas das questões a respeito da etiologia do craving e seus diferentes modelos explicativos, acabam por determinar a linha a ser utilizada, tanto em sua avaliação quanto em seu manejo.

Assim, os modelos comportamental, psicossocial ou cognitivo e biológico podem simplificar o significado de craving por meio de um paradigma reducionista e, conseqüentemente, delinear técnicas terapêuticas a partir de um único ponto de vista. Observa-se, na prática clínica e pelas pesquisas realizadas na área de dependência química, que as abordagens, como a terapia cognitivo-comportamental associada à farmacoterapia, que utilizam o modelo biopsicossocial, são as mais indicadas para o tratamento desta clientela, já que conseguem integrar os diversos modelos teóricos existentes. Utilizar recursos comportamentais, como o relaxamento e o recondicionamento, e cognitivos, como a reavaliação de crenças disparadoras de craving, somados ao apoio bioquímico pode, portanto, ser muito importante para auxiliar os dependentes químicos a manejar o craving.

Ao avaliar o craving, deve-se salientar que este é um fenômeno bastante dinâmico, à medida que a intensidade do desejo em um dado momento modifica-se após um curto período. Da mesma forma, estratégias que são utilizadas hoje com sucesso, talvez não tenham a mesma eficácia em outra situação.

A partir do aprofundamento do estudo do craving, pode-se concluir a favor de sua multidimensionalidade, a qual parece estar diretamente relacionada com a dificuldade que os indivíduos relatam ao tentar controlá-lo para manteremse em abstinência.

Analisando todos os aportes teóricos referentes a este construto, percebe-se o quanto o receio de avaliá-lo, de maneira fidedigna, pode atrapalhar o terapeuta na clínica. Por ser um estado subjetivo, a avaliação que o próprio sujeito tem de seu desejo, independentemente de estarem envolvidos processos cognitivos nem sempre conscientes, ainda é um instrumento muito valioso para orientar o tratamento. É importante ressaltar que o tratamento de dependentes químicos, em função das particularidades individuais, exige que terapeuta e cliente construam, juntos, o melhor projeto terapêutico e elejam as técnicas mais adequadas para cada caso, utilizando sempre a criatividade desta dupla ao pensar nas soluções para os dilemas da prática clínica.

\section{CONCLUSÃO}

Enfim, acredita-se que por causa de sua complexidade, o craving não é um conceito com o qual os pesquisadores e profissionais devam se acomodar. Ele suscita novos questionamentos, novas reflexões e a busca por diferentes estratégias para a sua avaliação e tratamento.

\section{REFERÊNCIAS}

1 Beck AT, Wright FD, Newman CF, Liese BS. Cognitive therapy of substance abuse. New York, NY: Gulford Press; 1993

2. Kozlowski LT, Pillitteri JL, Sweeney CT, Whitfield KE, Graham JW. Asking questions about urges or cravings for cigarettes. Psychology of Addict Behav. 1996;10(4):248-60.

3. Marques AC, Seibel SD. 0 craving. In: Seibel SD, Toscano Jr. A, editores. Dependência de drogas. São Paulo: Atheneu; 2001.

4. Sayette MA, Shiffman S, Tiffany S, Niaura R, Martin CS, Shadel W. The measurement of drug craving - methodological aproaches to craving research. Addiction. 2000;95 Suppl 2: S189-210.

5. Kozlowski LT, Wilkinson DA. Use and misuse of the concept of craving by alcohol, tobacco, and drug researchers. Br J Addiction 1987;82:31-6.

6. Singleton EG, Anderson LM, Heishman SJ. Reliability and validity of the tobacco craving questionnaire and validation of craving-induction procedure using multiple measures of craving and mood. Addiction. 2003;98:1537-46.

7. Tiffany ST. A cognitive model of drug urges and drug-use behavior: role of automatic and nonautomatic process. Psychol Rev. 1990;97:147-68.

8. United Nations International Drug Control Programme and World Health Organization (UNIDCP e WHO). Informal Expert Committe on Drug-Craving Mechanism (nº V. $92-54439$ T). Vienna, Report, 1992.

9. Araujo RB, Oliveira MS, Nunes MLT, Piccoloto LB, Melo WV. A avaliação do craving em alcoolistas na síndrome de abstinência. Psico-USF. 2004;9(1):71-6.

10. Tiffany ST, Drobes DJ. The development and initial validation of a questionnaire on smoking urges. Br J Addict. 1991;86:1467-76.

11. Knapp P, Luz Jr. E, Baldisserotto GV. Terapia cognitiva no tratamento da dependência química. In: Rangé B, editor. Psicoterapias cognitivo-comportamentais - um diálogo com a psiquiatria. Porto Alegre: Artes Médicas; 2001

12. Marlatt A, Gordon J. Prevenção de recaída - estratégias de manutenção no tratamento de comportamentos adictivos. Porto Alegre: Artes Médicas; 1993.

13. Cox LS, Tiffany ST, Christen AG. Evaluation of the brief questionnaire of smoking urges (QSU-brief) in laboratory and clinical settings. Nicotine Tob Res. 2001;3:7-16.

14. Carter BL, Tiffany ST. Meta-analysis of cue-reactivity in addiction research. Addiction. 1999;94: $327-40$.

15. Araujo RB, Oliveira MS, Piccoloto LB, Szupszynski KPDR. Sonhos e craving em alcoolistas na fase de desintoxicação. Rev Psiq Clin. 2004b;31(2):63-9.

16. Mahoney JJ, Kalechstein AD, De La Garza R, Newton TF. A qualitative and quantitative review of cocaine-induced craving: the phenomenon of priming. Prog Neuropsychopharmcol. Biol Psychiatry. 2007;31(3):593-9.

17. Brody AL, Mandelkern MA, London ED, Childress AR, Lee GS, Bota RG, et al. Brain metabolic changes during cigarette craving. Arch Gen Psychiatry. 2002;59(12):1162-72.

18. Hutchison KE, Lachance H, Niaura R, Bryan A, Smolen A. The DRD4 VNTR polymorphism influences reactivity to smoking cues. J Abnorm Psychol. 2002;111(1):134-43. 
19. Shiffman S, Shadel WG, Niaura R, Khayrallah MA, Jorenby DE, Ryan CF, Ferguson CL. Efficacy of acute administration of nicotine gum in relief of cue-provoked cigarette craving. Psychopharmacol. 12003;66(4):343-50.

20. Bandura A. Self-efficacy: toward a unifying theory of behavior change. Psychol Rev. 1977; 84(2):191-215.

21. Knapp P. Prevenção da recaída. In: Ramos S, Bortolote JM, editores. Alcoolismo hoje. Porto Alegre: Artes Médicas; 1997.

22. Gwaltney CJ, Shiffman S, Norman GJ, Paty JA, Kassel JD, Gnys M, et al. Does smoking abstinence self-efficacy vary across situations? Identifying context-specificity within the Relapse Situation Efficacy Questionnaire. J Consult Clin Psychol. 2001;69(3):516-27.

23. Niaura R, Shadel WG, Britt DM, Abrams DB. Response to social stress, urge to smoke, and smoking cessation. Addict Behav. 2002;27(2):241-50.

24. Ames SC, Roitzsch JC. The impact of minor stressful life events and social support on cravings: a study of inpatients receiving treatment for substance dependence. Addict Behav. 2000;25(4):539-47.

25. Erblich J, Boyarsky Y, Spring B, Niaura R, Bovbjerg DH. A family history of smoking predicts heightened levels of stress-induced cigarette craving. Addiction. 2003;98(5):657-64.

26. Reuter $M$, Netter $P$. The influence of personality on nicotine craving: a hierarchical multivariate statistical prediction model. Neuropsychobiology. 2001;44(1):47-53.

27. Gilbert HM, Warburton DM. Craving: A problematic concept in smoking research. Addict Res. 2000;8(4):381-97.

28. Sayette MA. Cognitive theory and research. In: Leonard KE; Blane HT. Psychological theories of drinking and alcoholism. The Guilford substance abuse series. New York: Guilford Press; 1999. p. 247-91.

29. Dijkstra A, Borland R. Residual outcome expectations and relapse in ex-smokers. Health Psychol. 2003;22(4):340-6.

30. Dols M, van den Hout M, Kindt M, Willems B. The urge to smoke depends on the expectation of smoking. Addiction. 2002;97:87-93.

31. Epping JM, Watkins SS, Koob GF, Markou A. Dramatic decreases in brain reward function during nicotine withdrawal. Nature-London. 1998;393(6680):76-9.

32. Niaura R, Goldstein MG, Ward KD, Abrams DB. Reasons for smoking and severity of residual nicotine withdrawal symptoms when using nicotine chewing gum. B J Addiction 1989;84(6):681-7.

33. Sayette MA, Martin CS, Hull JG, Wertz JM, Perrott MA. Effects of nicotine deprivation on craving response covariation in smokers. J Abnorm Psychol. 2003;112(1):110-8.

34. Reuter M, Netter P, Toll C, Henning J. Dopamine agonist and antagonist responders as related to types of nicotine craving and facets of extraversion. Prog Neuropsychopharmacol Biol Psychiatry. 2002;26(5):845-53.

35. Childress AR, Mozley PD, Mcelgin W, Fitzgerald J, Reivich M, O'brien CP. Limbic activation during cue-induced cocaine craving. Am J Psychiatr. 1999;156:11-8.

36. Branchey-Buydens L, Branchey M, Fergeson P, Hudson J, Mckernin C. Hormonal, psychological, and alcohol craving changes after m-chlorophenylpiperazine administration in alcoholics. Clin Exp Res. 1997;21(2):220-5.

37. Patkar AA, Gottheil E, Berrettini WH, Hill KP, Thornton CC, Weinstein SP. Relationship between platelet serotonin uptake sites and measures of impulsivity, agression, and craving among African-American cocaine abusers. Am J Addict. 2003;12(5):432-47.

38. Schroeder BE, Binzak JM, Kelley AE. A common profile of prefrontal cortical activation following exposure to nicotine or chocolate associated contextual cues. Neuroscience. 2001;105(3):535-45.

39. Anton R, Drobes DJ. Clinical measurement of craving addiction. Psychiatr Ann. 1998; 28(10):553-60
40. Anton RF, Moak DH, Latham P. The Obsessive compulsive drinking scale: a self-rated instrument for the quantification of thoughts about alcohol and drinking behavior. Alcohol Clin Exp Res. 1995;19(1):92-9.

41. Araujo RB, Oliveira MS, Mansur MA. A validação brasileira do questionnaire of smoking urges. Cad Saúde Pública. 2006;(10):2157-67.

42. Araujo RB, Oliveira MS, Moraes JFD, Pedroso RS, Port F, Castro MGT. Validação da versão brasileira do Questionnaire of Smoking Urges-Brief. Rev Psiq Clin. 2007;34(4):166-75.

43. Sayette MA, Parrott DJ. Effects of olfactory stimuli on urge reduction in smokers. Exp Clin Psychopharmacol. 1999;7(2):151-9.

44. Araujo RB. A relação entre sonhos e craving em alcoolistas na fase de desintoxicação [dissertação não publicada]. Porto Alegre: Pontifícia Universidade Católica do Rio Grande do Sul, Instituto de Psicologia, 2002.

45. Ussher M, Nunziata P, Cropley M, West R. Effect of a short bout of exercise on tobacco withdrawal symptoms and desire to smoke. Psychopharmacol. 2001;158(1):66-72.

46. Hernandez-Reif M, Field T, Hart S. Smoking cravings are reduced by self-massage. Prev Med. 1999;28(1):28-32.

47. Bobes J, Carreno JE, Gutierrez CE, San Narciso GI, Antuna MJ, et al. Study of effectiveness of craving control with topiramate in patients with substance dependence disorders. Actas Esp Psiquiatr. 2004;32(5):299-306.

48. Dale LC, Ebbert JO, Glover ED, Croghan IT, Schroeder DR, Severson HH, et al. Bupropion SR for the treatment of smokeless tobacco use. Drug Alcohol Depend. 2007;90(1):56-63.

49. Johnson BA, Roache JD, Bordnick OS, Ait-Daoud N. Isradipine, a dihydropyridine-class calcium channel antagonist, attenuates some of demethamphetamine's positive subjective effects: a preliminary study. Psychopharmacol. 1999;144(3):295-300

50. Kampman KM, Pettinati H, Lynch KG, Sparkman T, O'Brien CP. A pilot trial of olanzapine for the treatment of cocaine dependence. Drug Alcohol Depend. 2003;70(3):265-73.

51. Tonstad S, Tonnesen P, Hajek P, Williams KE, Billing CB, Reeves KR. Effect of maintenance therapy with varenicline on smoking cessation: a randomized controlled trial. JAMA. 2006;296(1):64-71

52. Volpicelli JR, Volpicelli JR, Rhines KC, Rhines IS, Volpicelli LA, Alterman Al, O'Brien CP. Naltrexone in the treatment of alcohol dependence. Arch Gen Psychiatry. 1992;49: 11:876-80.

53. Witters NW, Pulvirenti L, Koob GF, Gillin JC. Cocaine abuse and dependence. J Clin Psychopharmacol. 1995;15(1):63-78

54. Baker SL. The effect of nicotine and imaginal exposure to anxiety and smoking cues on smoking urge. Diss Abst Int Pt B Sci Eng. 2001;62(2-B):1065.

55. Campbell J, Nickel EJ, Penick EC, Wallace D, Gabrielli WF, Rowe C et al. Comparison of desipramine or carbamazepine to placebo for crack cocaine-dependent patients. Am J Addict. 2003;12(2):122-36.

56. Castro LA, Baltieri DA. Tratamento farmacológico da dependência do álcool. Rev Bras Psiq. 2004;26(supl.):43-6.

57. Durcan MJ, Deener G, White J, Johnston JA, Gonzales D, Niaura R et al. The effect of Bupropion sustained-release on cigarette craving after smoking cessation. Clin Ther. 2002;24(4):540-51.

58. Halikas JA, Crosby RD, Pearson VL, Graves NM. A randomized double-blind study of carbamazepine in the treatment of cocaine abuse. Clinical Pharmacol Ther. 1997; 62(1):89-105.

59. Johnson RE, McCagh JC. Buprenorphine and naloxone for heroin dependence. Curr Psychiatry Rep. 2000;2(6):519-26. 